\title{
Intravesical Anesthesia for Bladder Tissue Biopsies. Comparison of Two Methods
}

\author{
Vasileios G. Adamopoulos, Ioannis Filiadis, Erotokritos Konstandinidis
}

Department of Urology, General Hospital of Kavala, Kavala, Greece

\begin{abstract}
Purpose: To estimate the level of analgesia which can be obtained with simple intravesical instillation of ropivacaine in comparison to the combination of both instillation and subepithelial injection of the same agent.

Materials and Methods: Fifty-two patients were randomized in order that half (26) of them received simple intravesical instillation of ropivacaine $(100 \mathrm{~mL}$ solution of ropivacaine in a concentration of $2 \mathrm{mg} / \mathrm{mL})(\mathrm{Group} A)$, whereas the other 26 patients received both intravesical instillation and subepithelial injection of $2 \mathrm{~mL} \mathrm{(4} \mathrm{mg)} \mathrm{at} \mathrm{the} \mathrm{site} \mathrm{of} \mathrm{biopsy} \mathrm{(Group}$ B). In both groups, tissue samples were obtained from urinary bladder (number of biopsies from 3 to 4 ). The pain during the procedure was estimated by using the Visual Analogue Scale (VAS) which ranged from 0 to 10.

Results: The entire procedure was integrated with success in 50 out of 52 patients. The VAS score for the Group A ranged from 4 to 6 (mean 5.08), whereas for Group B from 1 to 3 (mean 1.6). ( $p<0.0001)$. Higher values of VAS score were recorded in males in both Groups $(\mathrm{p}<0.05)$. When complications of this method produced a slight bleeding (hematuria) in 6 patients ( 2 from group B and 4 from group A), they were treated with oral administration of fluids. Allergic reactions were not recorded. Hospitalization did not exceed 3 hours after the procedure.

Conclusions: The analgesic effect that was obtained with the combination of intravesical instillation and subepithelial injection of ropivacaine provides a safe method of anesthesia for transurethral bladder biopsy.
\end{abstract}

Key words: bladder, local anesthesia, ropivacaine, biopsy

Int Braz J Urol. 2008; 34: 277-82

\section{INTRODUCTION}

The biopsy of the urinary bladder is a minimally invasive procedure, which is commonly employed for detection of urinary bladder pathology (such as carcinoma in situ, interstitial cystitis, etc).

Several studies suggest that the analgesia, which can be obtained with intravesical instillation before transurethral biopsy of the urinary bladder, may offer an acceptable level of analgesia, although this procedure does not totally eliminate patient's complaints by using this method (1-5).
The aim of this pre-emptive study was to attempt to estimate the analgesic effect during the biopsy procedure, which may be administered by either the simple instillation of ropivacaine or the combined instillation and subepithelial injection of the same agent. A comparison between the two methods was accordingly performed.

\section{MATERIALS AND METHODS}

Fifty-two randomized patients (30 male, 22 female) with an age range 25-86 (mean 56.42) were 
enrolled in this study. All patients were fully informed regarding both the procedure and the type of analgesia and provided their written consent, which was formulated according to local legislation.

The patients were randomized so that $50 \%$ of them $(16 \mathrm{M}, 10 \mathrm{~F})$ received simple intravesical instillation of ropivacaine (Group A) whereas the other 26 patients $(14 \mathrm{M}, 12 \mathrm{~F})$ received both intravesical instillation and sub epithelial injection at the site of biopsy (Group B). Cup biopsies were performed for histopathologic examination of suspicious for nonexophytic neoplasia areas of bladder urothelium, mainly carcinoma in situ (CIS).

In each patient intraurethral lubrication with lidocaine hydrochloride $2 \%$ gel was performed before the procedure. Then, a 10F Nelaton catheter was used for intravesical instillation of $100 \mathrm{~mL}$ solution of ropivacaine $(2 \mathrm{mg} / \mathrm{mL}), 30$ minutes before the biopsy.

In the endoscopic operation room of our department a diagnostic cystoscopy was performed using a 24F rigid endoscope (Karl Storz). In the Group A, biopsies were obtained in a straightforward manner using grasp forceps. In the Group B a flexible metal needle (Karl Storz 27184A) was inserted through the working channel of the rigid endoscope. After the detection of the suspicious area, submucosa injection $2 \mathrm{~mL}$ of ropivacaine $2 \mathrm{mgr} / \mathrm{mL}$ was performed taking care to avoid bleeding of the area, which subsequently was biopsied with grasp forceps (cup biopsy). In both groups biopsies were from 3 to 4 .

Each patient was asked to estimate the severity of pain during the procedure using the Visual Analogue Scale (VAS) from 0 to 10 and remained in the Urology Department for a short-term observation.

The VAS values and the gender of each patient were recorded. Statistically significant differences $(\mathrm{p}<0.05)$ between the VAS values between the two Groups were analyzed based on the type of analgesia and the gender, using the Student's-t-test for independent samples (two tailed).

\section{RESULTS}

The entire procedure was integrated with success in 50 out of 52 patients. In 2 patients the pro- cedure was not completely performed due to poor endoscopic conditions (low visibility caused by prostate bleeding in the presence of enlarged prostate). One of these patients was in Group A and one in Group B.

The VAS score for the Group A ranged from 4 to 6 (mean 5.08), whereas for the Group B from 1 to 3 (mean 1.6) $(\mathrm{p}<0.0001)$. In each group (A and $\mathrm{B})$, the VAS values were significantly higher in men than in women of the same Group $(\mathrm{p}=0.0005$ and $\mathrm{p}<0.0001$, respectively) (Table-1). Statistically significant difference $(p<0.0001)$ was observed in VAS values depending on gender between the two groups (Table-1).

There was no systemic adverse effect from ropivacaine. Due to complications of this method, a slight bleeding (hematuria) occurred in 6 of 50 patients ( 2 from Group B and 4 from Group A), which was resolved using oral administration of water. Allergic reactions were not recorded. Hospitalization never exceeded 3 hours after the procedure.

\section{COMMENTS}

Minimally invasive techniques are employed in routine urological surgery with increasing frequency, possibly due to the demand for treating patients by one-day surgery. The employment of local anesthesia offers a large variety of benefits such as the patient's safety $(2,5,6)$, minimal hospitalization, the reduction of any complications associated with general or epidural anesthesia and the low cost $(4,6,7)$.

Although pain is a subjective symptom, the employment of the VAS score was used for the purpose of this study in order to estimate, as objectively as possible, this reaction in our patients (from slight discomfort to severe pain).

The intravesical instillation of a local anesthetic was initially described in $1991(1,2)$ and since then many authors have followed the same practice, as referred in many studies. Although the administration of lidocaine by EMDA technique offers effective anesthesia at the level of urothelium $(8,9)$, it is a rather sophisticated method. Intravesical instillation is safe, since the levels of Lidocaine are significantly much lower as regards the toxic levels, even if the technique is employed on denuded urothelium and almost totally 
Intravesical Anesthesia

Table 1 - Number of patients, visual analogue scale (VAS) values, mean VAS values and statistical differences between the groups.

\begin{tabular}{lcccc}
\hline & N & Sum - VAS & Mean - VAS & p Value (two tailed) \\
\hline Group A & 25 & 127 & 5.08 & $<0.0001$ \\
Group B & 25 & 40 & 1.6 & \\
Group A (men) & 16 & 87 & 5.43 & \\
Group A (women) & 9 & 40 & 4.44 & $<000563$ \\
Group B (men) & 14 & 29 & 2.07 & \\
Group B (women) & 11 & 11 & 1 & $<0.0001$ \\
Group A (men) & 16 & 87 & 5.43 & $<0.0001$ \\
Group B (men) & 14 & 29 & 2.07 & \\
Group A (women) & 9 & 40 & 4.44 & 1 \\
Group B (women) & 11 & 11 & & \\
\hline
\end{tabular}

harmless whenever the technique is employed on intact urothelium $(1-3,5,10)$. In addition to lidocaine, other local anesthetics such as bupivacaine have been employed either intravesically or as subtrigonal injection $(6,11,12)$ without any adverse effects.

Ropivacaine is the pure S (-) enantiomer of N-propyl-2'6-pipecoloxylidide and created from the need to produce a local anesthetic effective over a long period without any cardio toxicity which is, although very rarely the case, associated with bupivacaine. Ropivacaine prohibits both the initiation and the transmission of neural signals by reduction of membrane permeability of the neural cell in $\mathrm{Na}+$. The consequential arrest of depolarization leads to conductibility arrest. Small neural fibers are more sensitive to this effect and therefore demand a longer period of rehabilitation. The sensory fibers of pain are the first that are usually blocked. The extension of anesthesia depends on the diffusion of the solution, which is mostly affected by the area where this solution is administered and by the amount of the administered solution (13). To our knowledge, it is the first time that ropivacaine was employed for both local intravesical and submucosa anesthesia of the bladder. The concentration of $2 \mathrm{mg} / \mathrm{mL}$ was empirically selected in this study.

In addition, although the employment of the submucosa injection of a local anesthetic has proved efficacious for transurethral resection of superficial bladder tumors (6) and the intravesical instillation of similar agents have been used for minimal transurethral operations (14-16), this is the first time that a combination of both techniques was performed.

As clearly demonstrated in our study by the difference in VAS values between the two groups, the injection of ropivacaine by needle at the biopsy site combined with the intravesical instillation of the same agent offers greater reduction of pain than simple instillation of the same agent. Therefore, it is clearly suggested that the submucosa injection improves the analgesic effect of a local anesthetic, which was previously instilled into the urinary bladder. The superiority of the combined technique (intravesical instillation plus submucosa injection) as compared to simple instillation may be attributed to the prohibition 
of signals from sensory receptors to the centripetal C fibers, which form a submucosa network at the bladder wall. This network is responsible for activation of detrusor muscle. The action of local anesthetics, as has been proved recently, is not limited only to sensory fibers but extends to the centrifugal neural fibers and to the detrusor muscle, although this extended action demands higher concentrations (17).

Male patients experienced more pain than women possibly due to the differences in the anatomy of the lower urinary tract between two sexes, such as already mentioned by others (18).

Our method is easily applicable and safe. All cases were performed on an outpatient basis. The slight hematuria in a small number of patients subsided soon after the procedure and was mostly attributed to the effect of tissue resection from biopsy and not to the puncture of bladder wall for injecting the local anesthetic.

Furthermore, our results suggest that the analgesic effect of the combination of both submucosa injection and intravesical instillation (mean VAS value 1.6 in a 10-scale VAS) might be similar to the analgesic effect of caudal anesthesia during transurethral biopsies of urinary bladder with forceps (mean VAS value 0.8 in a 5 -scale VAS) (5). Therefore, if our observation is confirmed in a larger number of patients, any type of anesthesia could be replaced by this method, whenever there is a need for bladder biopsies or, at least, in patients who are at high risk for other types of anesthesia.

\section{CONCLUSIONS}

Transurethral biopsies of the urinary bladder can be performed with high level of analgesia whenever the combination of intravesical instillation and submucosa injection of ropivacaine is employed. The analgesic effect of this combination could be similar to caudal anesthesia and significantly superior as compared to simple instillation. Therefore, the intravesical instillation and the simultaneous submucosa injection of ropivacaine can safely replace caudal or general anesthesia and their subsequent morbidity when there is need for urinary bladder biopsies or any other minimal invasive transurethral procedure of the urinary bladder.

\section{CONFLICT OF INTEREST}

\author{
None declared.
}

\section{REFERENCES}

1. Thrasher JB, Peterson NE, Donatucci CF: Lidocaine as a topical anesthetic for bladder biopsies. J Urol. 1991; 145: 1209-10.

2. Pode D, Zylber-Katz E, Shapiro A: Intravesical lidocaine: topical anesthesia for bladder mucosal biopsies. J Urol. 1992; 148: 795-6.

3. Thrasher JB, Kreder KJ, Peterson NE, Donatucci CF: Lidocaine as topical anesthesia for bladder mappings and cold-cup biopsies. J Urol. 1993; 150: 335-6.

4. Holmäng S, Aldenborg F, Hedelin H: Multiple bladder biopsies under intravesical lignocaine anaesthesia. $\mathrm{Br}$ J Urol. 1994; 73: 160-3.

5. Amano T, Ohkawa M, Kunimi K, Oshinoya Y, Uchibayashi T: Topical anaesthesia for bladder biopsies and cautery: intravesical lidocaine versus caudal anaesthesia. Int Urol Nephrol. 1995; 27: 533-7.

6. Hedelin H, Holmäng S, Wiman L: Outpatient treatment of bladder cancer--lower cost and satisfied patients. Nord Med. 1997; 112: 48-51.

7. Holmäng S, Aldenborg F, Hedelin H: Extirpation and fulguration of multiple superficial bladder tumour recurrences under intravesical lignocaine anaesthesia. Br J Urol. 1994; 73: 177-80.

8. Fontanella UA, Rossi CA, Stephen RL: Bladder and urethral anaesthesia with electromotive drug administration (EMDA): a technique for invasive endoscopic procedures. Br J Urol. 1997; 79: 414-20.

9. Jewett MA, Valiquette L, Sampson HA, Katz J, Fradet Y, Redelmeier DA: Electromotive drug administration of lidocaine as an alternative anesthesia for transurethral surgery. J Urol. 1999; 161: 482-5.

10. Birch BR, Miller RA: Absorption characteristics of lignocaine following intravesical instillation. Scand J Urol Nephrol. 1994; 28: 359-64.

11. Westney OL, Lee JT, McGuire EJ, Palmer JL, Cespedes RD, Amundsen CL: Long-term results of Ingelman-Sundberg denervation procedure for urge 
incontinence refractory to medical therapy. J Urol. 2002; 168: 1044-7.

12. Matthews RD, Nolan JF, Libby-Straw JA, Sands JP Jr: Transurethral surgery using intravesical bupivacaine and intravenous sedation. J Urol. 1992; 148: 1475-6.

13. González T, Arias C, Caballero R, Moreno I, Delpón E, Tamargo J, et al.: Effects of levobupivacaine, ropivacaine and bupivacaine on HERG channels: stereoselective bupivacaine block. Br J Pharmacol. 2002; 137: 1269-79.

14. Dryhurst DJ, Fowler CG: Flexible cystodiathermy can be rendered painless by using $2 \%$ lignocaine solution to provide intravesical anaesthesia. BJU Int. 2001; 88: 437-8. Erratum in: BJU Int 2002; 89: 140.
15. Shackley DC, Briggs C, Gilhooley A, Whitehurst C, O'Flynn KJ, Betts CD, et al.: Photodynamic therapy for superficial bladder cancer under local anaesthetic. BJU Int. 2002; 89: 665-70.

16. Jønler M, Lund L, Bisballe S: Holmium: YAG laser vaporization of recurrent papillary tumours of the bladder under local anaesthesia. BJU Int. 2004; 94: 322-5.

17. Oh SJ, Kim SJ, Park EC, Chung HK, Kim KW, Choi $\mathrm{H}$ : Effects of local anesthetics on the contractility of rat bladders. J Urol. 2001; 165: 2044-50.

18. Taghizadeh AK, El Madani A, Gard PR, Li CY, Thomas PJ, Denyer SP: When does it hurt? Pain during flexible cystoscopy in men. Urol Int. 2006; 76: 301-3.

$\overline{\text { Accepted after revision: }}$ January 22, 2008

\section{Correspondence address:}

Dr. Vasileios G. Adamopoulos

Solomou 33-35

Heraklio, Crete, PC 713 06, Greece

E-mail: adamsbillgr@hotmail.com

\section{EDITORIAL COMMENT}

Transurethral biopsy of the bladder lesion is an office-based procedure. Although this procedure is minimally invasive and most of the patients can tolerate the bladder biopsy without any anesthesia, pain associated with the biopsy is still a matter that urologist should take into consideration. This study reported the results comparing the VAS responses of patients receiving intravesical and combined intravesical and suburothelial ropivacaine anesthesia for bladder mucosa biopsy. The results showed that combined anesthesia offers remarkable anesthetic action than intravesical anesthesia alone. A higher VAS score was reported in patients with intravesical anesthesia alone and in male patients of both procedures.
Recent investigations have shown that suburothelial space is rich in sensory nerve plexus. Many sensory receptors and neuromediators such as calcitonine-gene related peptides and nerve growth factor may contribute to the perception of bladder pain or activation of detrusor overactivity. Although intravesical instillation of anesthetic agent can provide anesthetic effect, the drug might not penetrate into the deep suburothelial space. Local anesthesia injects anesthetic agent into the deep suburothelial space and even the underlying muscle layer, which may eliminate bladder pain during biopsy procedure. Therefore, the results of this study seem to be expected because double anesthesia should be better than intravesical anesthesia alone. It will be interest- 
ing to know if intravesical anesthesia offers significant pain relief than no anesthesia, or local anesthesia with ropivacaine alone which has a similar effect when combined anesthesia. If local anesthesia alone is as effective as combined intravesical and local anesthesia, intravesical anesthesia might not be necessary in performing this minor bladder procedure.

Dr. Hann-Chorng Kuo Department of Urology Buddhist Tzu Chi General Hospital and Tzu Chi University Hualien, Taiwan E-mail:hck@tzuchi.com.tw

\section{EDITORIAL COMMENT}

In the management of superficial bladder cancer and carcinoma in situ, it is important to biopsy suspicious lesions in order to confirm or reject the existence of epithelial abnormalities. We have performed such procedures under general or spinal anesthesia where the patient was hospitalized. The authors have performed a randomized controlled study to investigate the efficacy of two different methods, intravesical instillation of ropivacaine and combination of both instillation and subepithelial injection of ropivacaine for patients who underwent a transurethral bladder biopsy in an outpatient basis and concluded that the combination is significantly better than the instillation alone.

As described by Taghizadeh et al. (references 18 in article), it has been demonstrated that the most painful part of flexible cystoscopy is when the tip of the cystoscope passes through the external sphincter. If it is compared in women only in this study in order to eliminate the effect of pain for lower urinary tract, it has been demonstrated that the combination is significantly better than the instillation alone however the number of patients in this study is small. Therefore, the combined intravesical and local anesthesia of ropivacaine for bladder biopsy might be able to replace caudal and general anesthesia with lower cost. However, this may be inadequate for lesions large enough to require resection rather than cold cup biopsy and those patients with poorly accessible regions of the bladder. Further studies are required to evaluate the cause of pain for bladder biopsy in order to perform better local anesthesia. 